\title{
Los mil días de oportunidad para intervenciones nutricionales. De la concepción a los dos años de vida*
}

\author{
The thousand days opportunity for nutritional interventions. \\ From conception to two years of life
}

\author{
Dr. César Victora ${ }^{a}$ \\ http:/ /dx.doi.org/10.5546/aap.2012.311
}

En esta conferencia abordaré el tema de los mil días, es decir, el dilema del crecimiento rápido: qué hacer y cuáles son las consecuencias del crecimiento rápido en niños pequeños.

$\mathrm{Al}$ respecto, puede haber consecuencias positivas y también algunas negativas.

Primero, corresponde establecer una diferencia, porque cuando se habla de crecimiento rápido se alude principalmente a la ganancia rápida de peso, pero no se habla de la talla. Se trata pues de parámetros distintos cuyos efectos también son distintos.

El otro aspecto importante, que se asocia con el crecimiento rápido, pero que no es la misma cosa, es el del crecimiento compensador (catch-up) después de un período de retraso de crecimiento (Cuadro 1). Se trata del niño que nació con bajo peso y crece más rápido, o que tuvo o tiene una serie de infecciones, de otros problemas, $y$ que después de un período de retraso se recupera.

Según los datos que existen en el mundo sobre el crecimiento rápido, cuando se los relaciona proporcionalmente con la superficie real del mundo, surgen ciertas distorsiones. Si se observa el total de nacimientos en el mundo, el mapa comienza a distorsionarse en África y el sur de Asia. Si se observa dónde están los estudios sobre las cohortes de nacimientos en los cuales basamos nuestras políticas, la relación es completamente distinta: en Finlandia o Inglaterra y Nueva Zelanda, por ejemplo, existen cohortes muy relevantes. Un punto esencial que deseo establecer en esta ponencia es que los estudios de las cohortes de nacimiento no se están llevando a cabo donde efectivamente ocurren la mayoría de los nacimientos y los resultados de estos estudios no necesariamente se pueden extrapolar.

En el Cuadro 2 se explica por qué se requieren más estudios en países de ingresos bajo y medio (PIBM), como

\section{Cuadro 1. Definiciones}

- Crecimiento rápido: ganancia rápida de peso en relación al promedio de la población.

- Catch-up: crecimiento rápido (compensador) después de un período de retraso de crecimiento.

Cuadro 2. ¿Por qué necesitamos estudios de cohorte en PIBM?

- Mayores frecuencias de algunas exposiciones (y menores frecuencias de otras).

- Diferentenaturaleza de algunas exposiciones.

- Diferentes patrones de factores de confusión.

- Diferencias sociales marcadas entre las cohortes de PIBM.

- Las cohortes de los PIBM están creciendo dentro de un contexto de transiciones epidemiológicas y nutricionales.

- El lugar de donde partimos puede afectar el resultado final.

PIBM: países de ingresos bajo y medio.
Correspondencia: cvictora@gmail.com

Conflicto de intereses: Ninguno que declarar.

Recibido: 10-5-2012 Aceptado: 10-5-2012
* Conferencia dictada en el Congreso del Centenario de la Sociedad Argentina de Pediatría. Buenos Aires, septiembre de 2011. 
es el caso de la mayoría de los de América del sur, Asia y África. El tema de las exposiciones es una cuestión fundamental para la epidemiología. Los epidemiólogos somos obcecados con la cuestión de los factores de confusión, y, cuando tenemos una asociación entre exposición y enfermedad, la primera pregunta que nos planteamos es si se trata de una relación de causa y efecto o si es debida a factores de confusión. Y esto es muy interesante, porque, por ejemplo, si se están estudiando los efectos de la lactancia en los países ricos en general, las mujeres ricas son las que más dan el pecho, amamantan durante más tiempo.

En los países pobres, muchas veces, es lo opuesto. Efectuar estas comparaciones es odioso porque los factores de confusión se mueven en direcciones opuestas según el tipo de país. Desafortunadamente, existen diferencias sociales muy marcadas en nuestras cohortes en países subdesarrollados, de ingresos bajo y medio. En las cohortes de países como Finlandia y Nueva Zelanda las diferencias sociales son muy pequeñas y esto también puede influenciar los resultados. Por otra parte, nuestros niños están creciendo en un momento de cambio, en un período de cambio muy rápido de transición nutricional. Las mismas generaciones que fueran subnutridas en la niñez ahora están obesas en la edad adulta. Se trata de un cuadro muy preocupante, pero que en términos de investigación científica también resulta muy estimulante para comprender mejor qué pasa en este proceso de transición. Y, finalmente, es bueno saber que el lugar desde donde partimos influencia el lugar al que llegamos (Cuadro 2). Tenemos una cohorte finlandesa donde el promedio de peso al nacer es de $3,5-3,6 \mathrm{~kg}$. No podemos extrapolar esos resultados para países cuyo promedio de peso al nacer puede ser de 3 o 2,9 kg, que son completamente distintos.

Para introducir este tema de los mil días, quiero referirme a unas investigaciones que realizamos hace más o menos quince años atrás. Yo sé que aquí, en la Argentina, hubo un debate acalorado sobre la adopción de las nuevas curvas de crecimiento. Con apoyo de la Organización Mundial de la Salud, en particular de la Dra. Mercedes de Onis, empezamos este estudio en Pelotas en 1997 y luego, con la colaboración de otros países, efectuamos un estudio en seis países distintos.

Creamos las curvas que se pueden ver en http:/ / www.who.int/childgrowth/standards/ Chap_4.pdf, páginas 95 y 123.

Como investigador, satisface constatar que más de cien países emplean las curvas que creamos hace algunos años. Incluso EE.UU., que es siempre renuente a adoptar lo que indica la comunidad internacional, porque allí prefieren tener sus propias curvas; ahora, finalmente, decidió emplearlas. Un sitio de California, en la ciudad de Davis, utiliza estas curvas como parte del estudio. Seguramente, las adoptaron porque también incluían por lo menos a un grupo de niños estadounidenses. Y lo más impresionante de estas curvas es que esos niños de tan alto nivel social, con lactancia materna, con madres que no eran fumadoras y que contaban con todas las características ideales de crecimiento, crecieron de manera muy similar a los de Brasil, India, Ghana, los Emiratos Árabes y Noruega.

Con esas curvas es posible realizar una serie de análisis. El año pasado publicamos el siguiente análisis: tomamos 54 encuestas nacionales demográficas donde se pesan niños de hasta cinco años (Figura 1). Son estudios transversales, con un solo examen en cada niño. Tomamos la curva de crecimiento y la graficamos como una recta plana. Entonces, la línea recta en el cero sería un niño que crece de acuerdo con las curvas. Sería una recta perfecta. ¿Qué observamos en esto? Observamos que el período en el cual los niños se distancian, que caen de la curva, finaliza a los 2 años de edad. La mayoría de estos estudios son realizados en Asia, África y en países pobres. A partir de los 2 años, tanto en peso como en talla, se mantienen más o menos paralelos a la curva. Con este tipo de constatación, también, observamos lo que llamamos la ventana de oportunidad. Aquí, en esta edad hasta los 2 años es que sucede el déficit de crecimiento (Figura 1). Claro que no es solamente

FIgURA 1. En PIBM, el crecimiento se ve afectado en los dos primeros años de vida

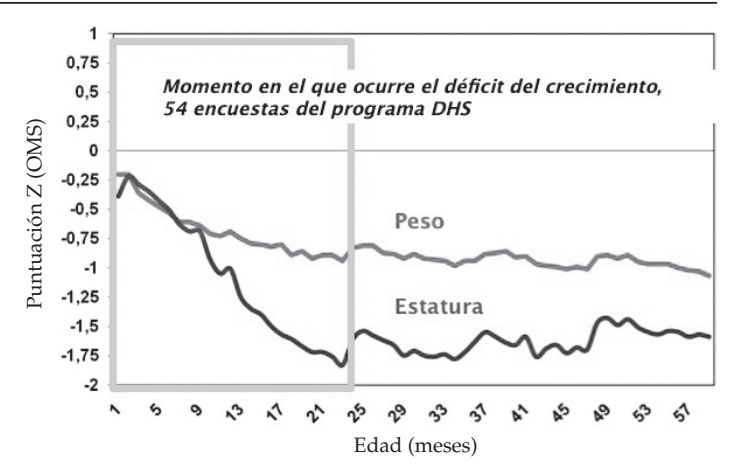

Victora et al, Pediatrics 2010

PIBM: países de ingresos bajo y medio. 
esto, el proceso empieza antes de la concepción, un poco antes de la concepción, como sabemos. $Y$, como es relevante hacer ciencia, pero también es importante emitir mensajes muy claros y sencillos, empezamos a hablar de los mil días, es decir, 270 días, más 365, más 365. Esto es un mensaje que se recuerda sin problemas.

Las consecuencias del retraso del crecimiento en los primeros años de vida son conocidas. En los estudios de los años setenta, ochenta, por ejemplo el caso del metaanálisis de David Pelletier y sus colegas de Cornell, básicamente, la mortalidad se vinculaba con el peso-edad. La relación es la siguiente: cuanto más alto el peso por edad, menos mueren los niños. Algo que, efectivamente, se sabe desde hace mucho tiempo. Pero el metaanálisis le da un carácter más científico.

El segundo mensaje de mi ponencia es que la subnutrición ocurre en los dos primeros años de vida, incluso en la gestación o en los mil dias, y que subsecuentemente aumenta la mortalidad. A corto plazo hay una oportunidad para intervenir en este grupo etario. Pero lo que más nos interesa al respecto en términos del conocimiento epidemiológico es que nos permite mirar qué pasará a largo plazo. Esto nos brinda la principal línea de investigación en la que está involucrado nuestro grupo de trabajo. Queremos saber qué sucede no sólo sobre la salud, sino también qué pasa con el capital humano. El concepto de capital humano es muy importante; supone hablar de inteligencia, de escolaridad, de talla, de fuerza muscular, de capacidad para generar hijos sanos en la próxima generación. En Pelotas, donde vivo, tenemos tres cohortes de niños estudiados.

Comenzamos con Fernando Barros en 1982, con una cohorte que seguimos hasta hoy y cuyo relevamiento de los treinta años vamos a efectuar el año que viene. Cuando la empezamos ni imaginábamos que, treinta años después, estaríamos en la Argentina hablando sobre la cohorte. Lo iniciamos como un estudio perinatal, que fue ganando interés. Luego comenzamos otra cohorte en 1993 y luego otra en 2004. Cada una registra todos los nacimientos de la ciudad de Pelotas en el año. Como se puede verificar por las estadísticas, cada vez tenemos menos niños en Brasil, la fecundidad en nuestro país está bajísima, mucho más baja que en la Argentina. Los nacimientos, para todas las clases sociales, se registran en la ciudad. Contamos con tasas de seguimiento que son bastante buenas. Con veinticinco años todavía seguimos al $75 \%$ de los seis mil que nacieron en 1982; en la cohorte de 1993 todavía tenemos
$88 \%$ y en la del 2004 registramos el $94 \%$ : altas tasas. Estas investigaciones de cohorte constituyen un enorme trabajo de seguimiento. Incluso algunos no viven más en Pelotas, se mudaron a otra ciudad. La descripción metodológica sobre estas tres cohortes ha sido publicada. ${ }^{1-3}$

Respecto de los análisis más recientes de las cohortes, sería muy interesante que se produjera una colaboración entre las mayores cohortes de países en desarrollo, justamente porque la mayoría de los resultados publicados en la bibliografía corresponden a los países ricos, y nosotros tenemos pocas cohortes. Por nuestra parte, identificamos las cinco mayores, las cohortes de más larga duración, en países en desarrollo. En el año 2006 se realizó una invitación a las cohortes de Guatemala, de Sudáfrica, a una de Nueva Delhi y a otra de Filipinas. Cada una representa, más o menos, a un continente diferente, a una parte distinta del mundo, lo cual es muy bueno para comparar los resultados. Así, se creó el consorcio que denominamos COHORTS (Consortium of Health Orientated Research in Transitioning Societies: Consorcio de Investigación Orientada a la Salud de Sociedades en Transición). ${ }^{4}$

Uno de los primeros análisis que realizamos fue comparar los resultados de las cinco cohortes como si se tratara de un metaanálisis. Respecto de la talla del adulto en relación con la talla-edad a los 2 años de edad (Figura 2), es muy interesante observar que, para un determinado puntaje $Z$ a los 2 años, en todos los países y para las distintas cohortes de varones y mujeres, los resultados fueron muy similares cuando adultos. Si tenían un puntaje $Z$ igual a $1(3,2 \mathrm{~cm})$ de talla-edad a los 2 años, tenían igualmente $3,2 \mathrm{~cm}$ más como adultos. Lo más impactante para mí fue que no lo creí

Figura 2. Altura del adulto según la puntuación Z de talla/ edad a los 2 años

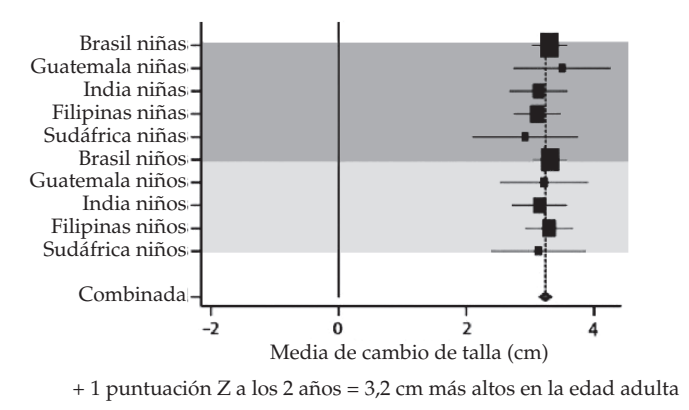

Victora et al., Lancet 2008 Ajustado para factores de confusión 
cuando observé los resultados. Primero, que los resultados fuesen tan homogéneos en lugares tan distintos como Brasil, África e India. Segundo, que el valor en centímetros de una puntuación $\mathrm{Z}$ a los 2 años fuese exactamente de 3,2 cm. Si en un niño, el promedio es $3 \mathrm{~cm}$ más alto a los 2 años, como adulto el promedio va a ser $3 \mathrm{~cm}$ más alto que el promedio de la población. Algo realmente muy impresionante.

En mi vida de epidemiólogo nunca he visto un metaanálisis tan uniforme, tan regular como éste. La talla es un indicador de capital humano, pero la escolaridad también lo es. Los sistemas educacionales de estos países son completamente distintos, pero los resultados vuelven a ser muy similares. Una puntuación $Z$, de talla-edad, en los 2 años se asocia con medio año más de escolaridad en la edad adulta. Esto controlado para un cúmulo de factores de confusión; para ingreso familiar, ocupación de los padres o por muchas otras variables distintas. A pesar de la diversidad, tenemos una asociación muy clara de la talla a los 2 años, respecto de lo que pasa con la escolaridad. Medio año de escolaridad no es poco. En muchas de estas sociedades, el promedio de escolaridad es de 4-5 años. Se trata de una diferencia significativa, incluso en términos de ingresos familiares.

Asimismo, como tenemos más de una generación en las cohortes actuales, podemos observar qué pasa con el peso al nacer de los niños de las mujeres que estudiamos desde el nacimiento; en la Figura 3 se observa que, en las cuatro cohortes donde registramos datos confiables, el puntaje de talla-edad a los 2 años se asocia con $80 \mathrm{~g}$ más de peso al nacer en la próxima generación. Si invertimos en nutrición, estamos realizando una inversión transgeneracional que se prolonga a largo plazo. Invertir en nutrición es una inversión en el

Figura 3. Peso al nacer (PN) de la siguiente generación según el puntaje $Z$ de talla/edad a los 2 años

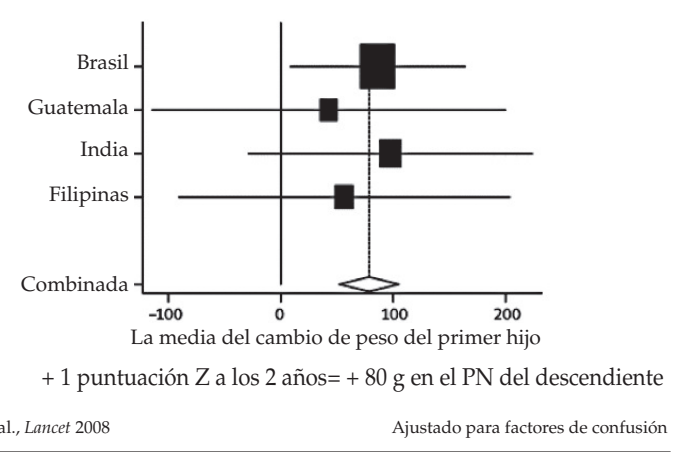

desarrollo del país. No se trata de una inversión especulativa, sino de una que supone beneficios a largo plazo. Para concluir solo resta decir que el mensaje es muy claro. Una buena nutrición en los primeros años de vida mejora: a) el capital humano y b) distintos indicadores poblacionales (de talla, escolaridad, peso de la próxima generación e, incluso, de ingresos y bienes). En individuos que ya los tienen más altos, aun controlados por factores de confusión, son igualmente más elevados.

Pero, ¿qué sucede cuando se produce la subnutrición? Es importante saber qué pasa con los efectos a largo plazo de la subnutrición y también cuándo se produce. Lo que hacemos en las cohortes es trabajar con la ganancia de peso en diferentes grupos etarios. Porque sus pronósticos difieren respecto de lo que pasará en la edad adulta. En la mayoría de los gráficos, el eje horizontal corresponde a la edad y el eje vertical a un desenlace, a un resultado (outcome), que puede ser bueno o malo. El caso aquí, en capital humano muestra resultados positivos (Figura 4). Nosotros trabajamos con pesos condicionales (conditional weights). Se trata de una técnica estadística que permite separar una ganancia de peso en cada intervalo de edad, que evita el problema de que ganancias en distintos grupos de edad estén correlacionados; es un método de regresión. En verdad, esta regresión se debe interpretar como lo que sucede con la trayectoria de un niño dado. Un peso condicional alto significa que dicho niño crecía de forma acelerada. Un peso condicional bajo indica que, en relación a lo que crecía antes, su crecimiento se ha desacelerado. Trabajamos, más o menos, con aceleración de crecimiento, que es la técnica de pesos condicionales, de regresión, que tiene en cuenta la ganancia anterior, en edades más tempranas. Ahora bien, qué sucede con la

FIgURA 4. Hallazgos sobre el capital humano (Pelotas, Brasil)

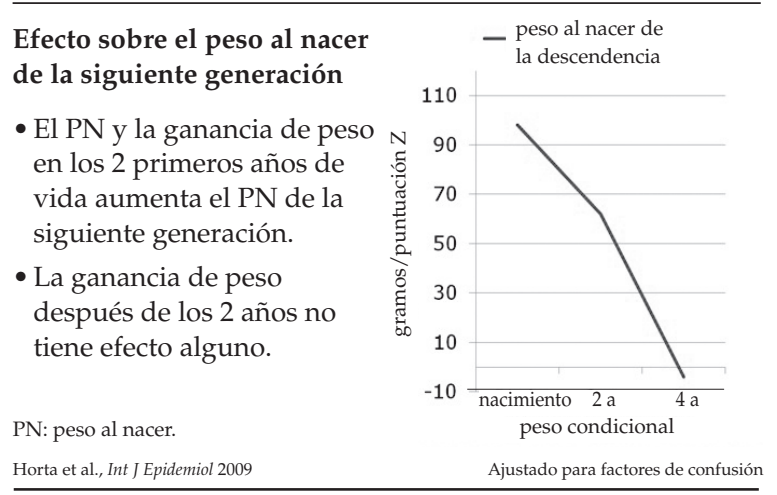


escolaridad; el peso al nacer tiene una puntuación $\mathrm{Z}$, este puntaje $\mathrm{Z}$ al nacer aumenta la escolaridad en, más o menos, 0,2 años. Si uno crece rápido hasta los 2 años de vida, el guarismo aumenta. $Y$ un crecimiento rápido desde que uno nace hasta los 2 años de vida aumenta la escolaridad en 0,45 años. Pero ganar peso rápidamente desde los dos hasta los cuatro años de vida no contribuye en nada. Esto tiene mucho sentido pues sabemos que el cerebro se desarrolla intraútero y en los primeros años de la vida, y que luego no crece mucho. Los estudios de las cinco cohortes coordinadas mostraron que aumentar de peso en los dos primeros años de vida tiene mucho sentido, que acelerar el crecimiento en los dos primeros años de vida ejerce un efecto positivo, pero que después de los 2 años no tiene efecto alguno, que no sirve de nada.

¿Cuál es la situación respecto del peso al nacer de la próxima generación? Resulta claro que el peso al nacer de la madre está fuertemente asociado con el peso al nacer del hijo, pues existen factores genéticos, no sólo nutricionales. Si la madre aumenta de peso rápidamente hasta los 2 años, también se incrementa en cerca de $60 \mathrm{~g}$ el peso del niño, pero si el aumento de peso se produce después de los 2 años, eso no tendrá efecto alguno sobre el peso de su niño, en la próxima generación. Nuevamente, queda clara la relevancia del lapso hasta los 2 años como el período crítico, donde las cosas realmente cambian.

La preocupación que surge en la bibliografía reciente es que la ganancia de peso puede ser muy buena para indicadores como la escolaridad, el capital humano, etc., pero existe mayor riesgo de enfermedades futuras, de enfermedades crónicas. Contamos con muchísimos resultados, principalmente de las cohortes en países ricos, que arrojan mapas distorsionados. Pero muchas de esas cohortes se conforman en países donde, a lo sumo, el bajo peso al nacer es del $4 \%, 6 \%$ u $8 \%$. Pero lo importante es saber qué pasa en los países donde existe un $10 \%, 15 \%$ o $20 \%$ de bajo peso. Aquí se presenta el problema de "traducir" los resultados de los países ricos a los países pobres o a los de ingresos medios. En tal contexto, es donde surgen en la bibliografía una serie de artículos que principalmente dicen que ganar peso rápidamente es malo. Un gran problema de esos artículos, por lo menos de la primera generación de ellos, es que normalmente sopesaban un peso al nacer y un peso a los 7 años (al ingreso escolar). Las cohortes de países desarrollados, en su mayoría, abundan en datos secundarios debido a que cuentan con sistemas muy buenos, donde todo está computa- rizado, no como donde se carece de los avances tecnológicos y es menester salir puerta a puerta y correr detrás de una persona. Dichos artículos muestran que, si el peso al nacer es bajo y se gana mucho peso a los 7 años, el riesgo de tener enfermedades crónicas es alto. Es verdad, pero lo que ha hecho nuestro grupo es definir esta ganancia de peso en distintos grupos de edad.

¿Qué pasa con el índice de masa corporal? Los análisis realizados permiten separar no solo los períodos de ganancia de peso, sino también la masa corporal magra y la masa corporal grasa. Cuando uno crece más rápido va a tener más masa corporal grasa, de eso no hay duda. Pero el efecto es relativamente pequeño para el peso al nacer, un poco más a los 2 años, a los 4 años, y, principalmente después de los 4 años, la curva de masa grasa responde más a la ganancia de peso. Para la masa magra es distinto (Figura 5). Si el niño crece rápido hasta los 2 años está adquiriendo más masa magra que masa grasa; después se invierte la situación. Esto es determinante, porque la masa magra significa músculos, huesos, vísceras y icerebro! Es importante que haya una inversión entre los dos y cuatro años en esta ganancia.

Para diabetes, desafortunadamente no tenemos en todos los estudios un diagnóstico extremadamente preciso, pero asimismo los hallazgos son interesantes. Al nacer, el ganar peso hasta los 2 años es protector, pero si se gana peso luego de los 4 años o más, aumenta el riesgo. La ganancia de peso inicial protege contra la diabetes, pero después pasa a ser un factor perjudicial. Igualmente, para la hipertensión el peso al nacer es ligeramente protector. Después aumenta levemente, en especial en la ganancia de peso después de los 4 años.

Figura 5. Hallazgos sobre los factores de riesgo para enfermedades crónicas

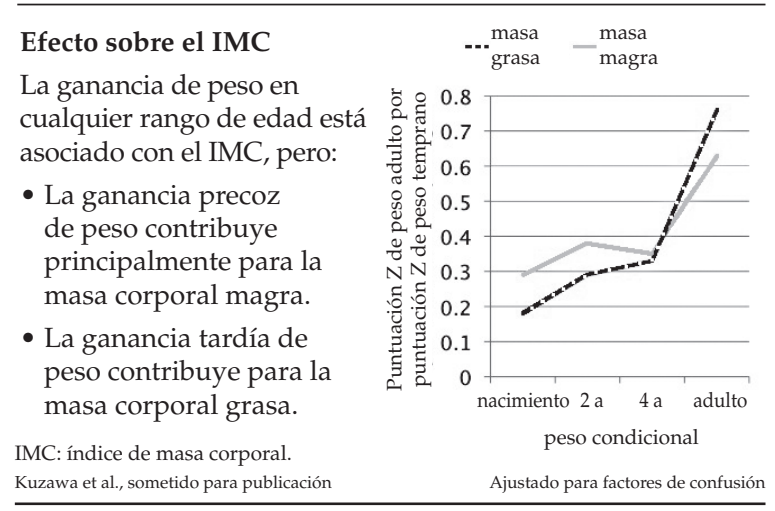


La proteína C-reactiva es un gran indicador de riesgo cardiovascular, para el cual tenemos datos de la cohorte de Pelotas. No todas las cohortes estudian la proteína C-reactiva. Nuestra cohorte la registra a los 24 años de edad. Después se observa el mismo patrón. El peso hasta un año es protector, luego se incrementa un poco, y principalmente más tarde aumenta el riesgo.

Por lo tanto, el cuarto mensaje de esta ponencia es que el momento en que ocurre la ganancia de peso es determinante, porque afecta el riesgo futuro para enfermedades crónicas. La ganancia precoz intraútero, o en el primero y segundo años de vida, tiene un efecto neutro o incluso protector contra algunas enfermedades. La ganancia de peso después de los 2 años ya ejerce efectos predominantemente perjudiciales para la salud en términos de factores de riesgo a largo plazo. Se trata de una definición muy importante.

En Guatemala se pudo observar muy bien la transición nutricional. Se trata de una población subnutrida la cual está ahora expuesta a comida chatarra (Mac Donalds, Dunkin Donuts, etc.) y a otros productos de ese tipo. ¿Qué sucede con esos grupos subnutridos que luego se tornan adultos obesos? En el estudio de la cohorte de Nueva Delhi, India ${ }^{5}$ la línea cero representa el crecimiento promedio de la población. Por ende, un individuo que estuviera en la línea cero estaría en el promedio. Los individuos que desarrollan intolerancia a la glucosa, o diabetes, en ese peso, están debajo de la curva, subnutridos, y luego ganan peso rápidamente. Esos son los que desarrollan diabetes. Este estudio es muy famoso. Al observar nuestras cohortes de Pelotas, regresamos a la proteína C-reactiva, y separamos a los niños con retraso de crecimiento intrauterino y a los sin él. Para los individuos sin restricción de crecimiento intrauterino existe un pequeño aumento de la proteína C-reactiva, pero en los que hubo retraso de crecimiento, la ganancia hasta los 2 años es protectora: la razón está debajo de uno. Ahora bien, después de los 2 años aumenta exponencialmente. Existe un período en cual el crecimiento compensador todavía protege, pero un crecimiento compensador tardío, es perjudicial. Y lo mismo se verifica para el perímetro abdominal, que también sabemos es un factor relevante asociado con el riesgo cardiovascular. Los niños sin retraso tienen una asociación más o menos baja en la ganancia de peso. Pero quienes tuvieran retraso intrauterino, si ganan peso después de los 2 años, aumentan mucho el riesgo de enfermedades futuras.
El último mensaje que deseo transmitir es que la peor combinación parece ser una subnutrición precoz, ya sea el bajo peso al nacer o el retraso de crecimiento intrauterino, y una rápida ganancia tardía de peso, no temprana. La ganancia precoz todavía compensa un poco la subnutrición, pero no la ganancia tardía en el individuo, que ya estaba programado.

Aquí, corresponde abordar el concepto de la programación, que nos parece una teoría cautivante. Actualmente entendemos mejor que lo relevante no es solo lo que pasa en el útero, sino también lo que sucede en los dos primeros años de vida. Qué es lo que programa a un individuo para ser pequeño mediante una serie de mecanismos neuroendocrinos. Esto lleva al hecho innegable de que lo que pasa en los primeros mil días repercute permanentemente sobre la salud del individuo. Para simplificar, aunque siempre resulta un poco peligroso simplificar, si queremos dirigirnos en lenguaje no técnico a la población (Figura 6), veamos qué pasa con la línea gris y qué con la línea negra. La línea gris es el capital humano y la mortalidad a corto plazo y la negra el riesgo de enfermedades crónicas. Si uno gana peso condicional, gana aceleración en el peso, esto es bueno para el capital humano, más aún en el inicio de la vida. Pero después de una cierta edad, prácticamente no se obtiene beneficio.

Para las enfermedades crónicas, ganar peso rápidamente o acelerar la trayectoria en los primeros 2 años de vida o en la fase intrauterina no aumenta el riesgo, pero, en países de ingresos bajos, solamente a partir de los dos o tres años empieza a aumentar el riesgo. ¿Por qué resulta importante en países de ingresos bajos?, porque en los países ricos es distinto. En los países ricos la

Figura 6. Ganancia de peso precoz en países de ingresos bajos

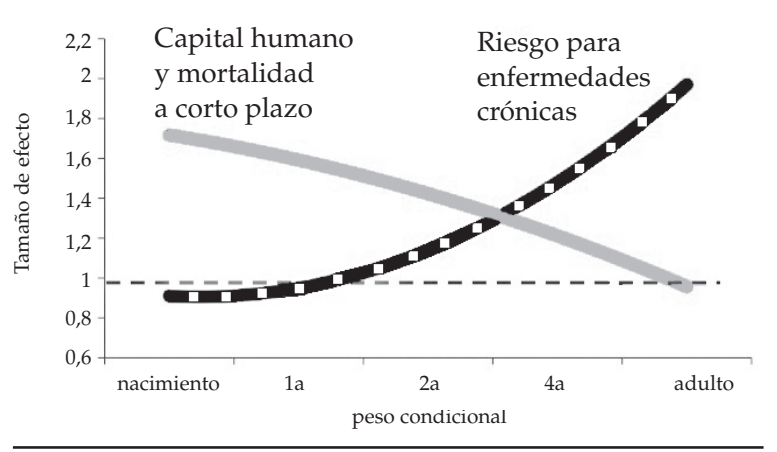


mortalidad es baja, los niños nacen con buen peso. Tener cohortes de nacimiento en países como Finlandia, Inglaterra, Nueva Zelanda, no es tan útil para nosotros, porque nuestras realidades son distintas y las poblaciones tampoco son las mismas.

Para concluir, el mensaje principal es destacar el enorme desafío para la salud pública que representa brindar atención a poblaciones de ingresos bajo y medio. Enfrentamos dos mensajes aparentemente contradictorios. En el primero de ellos indicamos que, en los mil días de oportunidad, es menester prevenir el déficit de crecimiento y hacer todo lo posible para que el niño crezca. Pero después de una cierta edad, de más o menos entre dos o tres años, debemos prevenir la ganancia muy rápida. Y eso, en términos poblacionales, no es precisamente un mensaje muy simple. Es algo bastante complejo como para asesorar a una madre o a quienes se desempeñan en la clínica o trabajan en salud pública. Debemos discutir mejor cómo operar en conjunto y modificar la manera en que trabajamos, asunto que me preocupa mucho. No soy pediatra, sino epidemiólogo. Antes de hacer epidemiología, cuando era médico de familia, siempre me enseñaron que a un niño de 3-4 años, con un peso para la edad bajo, había que hacerle ganar peso rápidamente. Pero el tipo de información que surge ahora de estas cohortes es que esto no es tan simple ni seguro. Después de una cierta edad podemos estar solamente contribuyendo con el riesgo de enfermedades crónicas, sin tener una ventaja sobre el capital humano, porque la ventana de oportunidad de los mil días ya está cerrada.

Para concluir, deseo expresar que este trabajo es colectivo y está aportando resultados muy interesantes. Representamos a un grupo de cinco cohortes que operan mancomunadamente desde hace muchos años. Todos nuestros trabajos están a disposición en nuestro sitio web (www.epidemio-ufpel.org.br).

\section{BIBLIOGRAFÍA}

1. Victora CG, Barros F C. Cohort profile: The 1982 Pelotas (Brazil) birth cohort study. Int J Epidemiol 2006;35(2):237-42.

2. Victora C G, Hallal PC, Araújo CL, Menezes AM, et al. Cohort profile: The 1993 Pelotas (Brazil) birth cohort study. Int J Epidemiol 2008;37:704-9.

3. Santos IS, Barros AJ, Matijasevich A, Domíngues MR, et al. Cohort Profile: The 2004 Pelotas (Brazil) birth cohort study. Int J Epidemiol 2011;40(6):1461-8.

4. Richter L.M, Victora CG, Hallal PC, Adair LS, et al. Cohort profile: the consortium of health-orientated research in transitioning societies. Int J Epidemiol 2011. Apr 21.Págs.1-6.

5. Bhargava SK, Sachdev HS, et al. Relation of serial changes in childhood body-mass index to impaired glucose tolerance in young adulthood. N Engl J Med 2004;350(9):865-75. 